\title{
HOPF BIFURCATION IN THE FULL REPRESSILATOR EQUATIONS
}

\author{
CLAUDIO A. BUZZI ${ }^{1}$ AND JAUME LLIBRE ${ }^{2}$
}

\begin{abstract}
In this paper we prove that the full repressilator equations, in dimension six undergo a supercritical Hopf bifurcation.
\end{abstract}

\section{INTRODUCTION}

Oscillatory networks are a particular kind of regulatory molecular networks, i.e., collections of interacting molecules in a cell. The regulatory oscillators can be used to study abnormalities of a process in the cell, from sleep disorders to cancer. So, they attract significant attention among biologists and biophysicists. There are many implementations of artificial oscillatory networks (see, e.g., $[1,2,6,7,8,10,11]$ ). One of them is the repressilator [5]. Its genetic implementation uses three proteins that cyclically repress the synthesis of one another. The following system of differential equations describes the behavior of the repressilator:

$$
\begin{aligned}
& \dot{m}_{1}=-m_{1}+\frac{\alpha}{1+v^{n}}+\alpha_{0}, \\
& \dot{m}_{2}=-m_{2}+\frac{\alpha}{1+w^{n}}+\alpha_{0}, \\
& \dot{m}_{3}=-m_{3}+\frac{\alpha}{1+u^{n}}+\alpha_{0}, \\
& \dot{u}=-\beta\left(u-m_{1}\right), \\
& \dot{v}=-\beta\left(v-m_{2}\right), \\
& \dot{w}=-\beta\left(w-m_{3}\right) .
\end{aligned}
$$

Here $u, v$ and $w$ are proportional to the protein concentration, while $m_{i}$ are proportional to the concentration of mRNA corresponding to those proteins. The nonlinear function $f(x)=\frac{\alpha}{1+x^{n}}$ reflects synthesis of the mRNAs from the DNA controlled by regulatory elements. The parameter $\alpha_{0}$ represents uncontrolled part of the mRNA synthesis, and it is usually small. The

2010 Mathematics Subject Classification. Primary

Key words and phrases. Limit cycles, oscillatory regulatory networks, repressilator. 
explicit inclusion of the mRNA concentration variables into the model is given by $\beta$. Given that in general $\beta \ll 1$ and $\alpha_{0}$ is very small, we consider $\alpha_{0}=\varepsilon a$ and $\beta=\varepsilon b$, where $a$ and $b$ are positive constants and $\varepsilon>0$ is sufficiently small. So, system (1) becomes

$$
\begin{aligned}
& \dot{m}_{1}=-m_{1}+\frac{\alpha}{1+v^{n}}+\varepsilon a, \\
& \dot{m}_{2}=-m_{2}+\frac{\alpha}{1+w^{n}}+\varepsilon a, \\
& \dot{m}_{3}=-m_{3}+\frac{\alpha}{1+u^{n}}+\varepsilon a, \\
& \dot{u}=-\varepsilon b\left(u-m_{1}\right), \\
& \dot{v}=-\varepsilon b\left(v-m_{2}\right), \\
& \dot{w}=-\varepsilon b\left(w-m_{3}\right) .
\end{aligned}
$$

In the papers $[3,4]$ the authors consider a reduced system of dimension three. The reduction assumes that the three excluded variables, i.e. $m_{i}$, evolve an order of magnitude faster than the other three. In [3] the authors prove that in the reduced system exhibits a supercritical Hopf bifurcation. The existence of a Hopf bifurcation in the reduced system does not imply that the full system, in dimension six, also has a supercritical Hopf bifurcation. It just gives an indication about its existence. Here in this work we consider the full system in dimension six and we extend the results of $[3,4]$ on the supercritical Hopf bifurcation to the 6 -dimensional differential system (2). Our main result is the following one.

Theorem 1. Let $n>2$ be an integer. The point $\left(r_{0}, r_{0}, r_{0}, r_{0}, r_{0}, r_{0}\right)$ with

$$
r_{0}=\sqrt[n]{\frac{2}{n-2}}+\varepsilon \frac{a-3 b \sqrt[n]{\frac{2}{n-2}}}{n-2}+\mathcal{O}\left(\varepsilon^{2}\right)
$$

is an equilibrium of the differential system (2) with

$$
\alpha=\alpha_{b i f}=\frac{n}{n-2} \sqrt[n]{\frac{2}{n-2}}+\varepsilon \frac{n}{(n-2)^{2}}\left(-a(n-5)-9 b \sqrt[n]{\frac{2}{n-2}}\right)+\mathcal{O}\left(\varepsilon^{2}\right) .
$$

The eigenvalues of the linear part of system (2) at this equilibrium are $\left\{ \pm \varepsilon \sqrt{3} b i+\mathcal{O}\left(\varepsilon^{2}\right),-1+\varepsilon(-1 \pm \sqrt{3} i) b+\mathcal{O}\left(\varepsilon^{2}\right),-\varepsilon 3 b+\mathcal{O}\left(\varepsilon^{2}\right),-1+\varepsilon 2 b+\mathcal{O}\left(\varepsilon^{2}\right)\right\}$. Moreover, there is a single supercritical Hopf bifurcation at $\alpha=\alpha_{b i f}$ and there exists a small $\varepsilon_{0}>0$ such that for $\alpha_{b i f}<\alpha<\alpha_{b i f}+\varepsilon_{0}$ the system (2) has a stable limit cycle. 


\section{Proof of Theorem 1}

It is clear that system (2) has the equilibrium $p_{0}=\left(r_{0}, r_{0}, r_{0}, r_{0}, r_{0}, r_{0}\right)$ where $r_{0}$ is solution of the equation

$$
\frac{\alpha}{1+r^{n}}=r-\varepsilon a \text {. }
$$

From (3) we have that $\alpha=\left(r_{0}-\varepsilon a\right)\left(1+r_{0}^{n}\right)$. So, substituting $\alpha$ in the linear part of system (2) at the equilibrium $p_{0}$ we get

$$
A=\left(\begin{array}{cccccc}
-1 & 0 & 0 & 0 & \Delta & 0 \\
0 & -1 & 0 & 0 & 0 & \Delta \\
0 & 0 & -1 & \Delta & 0 & 0 \\
\varepsilon b & 0 & 0 & -\varepsilon b & 0 & 0 \\
0 & \varepsilon b & 0 & 0 & -\varepsilon b & 0 \\
0 & 0 & \varepsilon b & 0 & 0 & -\varepsilon b
\end{array}\right),
$$

where $\Delta=-\frac{n r_{0}^{-1+n}\left(r_{0}-\varepsilon a\right)}{1+r_{0}^{n}}$. The eigenvalues of $M$ are

$$
\begin{array}{ll}
\varepsilon \frac{\left(-2+(n-2 \pm i \sqrt{3} n) r_{0}^{n}\right) b}{2\left(1+r_{0}^{n}\right)}+\mathcal{O}\left(\varepsilon^{2}\right), & -1+\varepsilon \frac{(-1 \pm i \sqrt{3}) n r_{0}^{n} b}{2\left(1+r_{0}^{n}\right)}+\mathcal{O}\left(\varepsilon^{2}\right), \\
-1+\varepsilon \frac{n r_{0}^{n} b}{1+r_{0}^{n}}+\mathcal{O}\left(\varepsilon^{2}\right), & -\varepsilon \frac{\left(1+(1+n) r_{0}^{n}\right) b}{1+r_{0}^{n}}+\mathcal{O}\left(\varepsilon^{2}\right) .
\end{array}
$$

We impose that the real part of the eigenvalues $\varepsilon \frac{\left(-2+(n-2 \pm i \sqrt{3} n) r_{0}^{n}\right) b}{2\left(1+r_{0}^{n}\right)}+$ $\mathcal{O}\left(\varepsilon^{2}\right)$ is zero and we obtain

$$
r_{0}=\sqrt[n]{\frac{2}{n-2}}+\varepsilon \frac{a-3 b \sqrt[n]{\frac{2}{n-2}}}{n-2}+\mathcal{O}\left(\varepsilon^{2}\right)
$$

Substituting (4) in (3) we get

$$
\alpha_{b i f}=\frac{n}{n-2} \sqrt[n]{\frac{2}{n-2}}-\varepsilon \frac{n}{(n-2)^{2}}\left(a(n-5)+9 b \sqrt[n]{\frac{2}{n-2}}\right)+\mathcal{O}\left(\varepsilon^{2}\right)
$$

Substituting (4) in $M$ and computing the eigenvalues we obtain $\pm \varepsilon \sqrt{3} b i+$ $\mathcal{O}\left(\varepsilon^{2}\right),-1+\varepsilon(-1 \pm \sqrt{3} i) b+\mathcal{O}\left(\varepsilon^{2}\right),-\varepsilon 3 b+\mathcal{O}\left(\varepsilon^{2}\right),-1+\varepsilon 2 b+\mathcal{O}\left(\varepsilon^{2}\right)$.

The linearization of $(2)$ at $p_{0}$ has a pair of conjugate purely imaginary eigenvalues and the other four eigenvalues have negative real part. This is the setting for a Hopf bifurcation. We can expect to see a small-amplitude 
limit cycle branching from the fixed point $p_{0}$. It remains to compute the first Lyapunov coefficient $\ell_{1}\left(p_{0}\right)$ of $(2)$ near $p_{0}$. When $\ell_{1}\left(p_{0}\right)<0$ the point $p_{0}$ is a weak focus of system (2) restricted to the central manifold of $p_{0}$ and the limit cycle that emerges from $p_{0}$ is stable. In this case we say that the Hopf bifurcation is supercritical. When $\ell_{1}\left(p_{0}\right)>0$ the point $p_{0}$ is also a weak focus of system (2) restricted to the central manifold of $p_{0}$ but the limit cycle that borns from $p_{0}$ is unstable. In this second case we say that the Hopf bifurcation is subcritical.

Here we use the following result presented on page 180 of the book [9] for computing $\ell_{1}\left(p_{0}\right)$.

Lemma 2. Let $\dot{x}=F(x)$ be a differential system having $p_{0}$ as an equilibrium point. Consider the third order Taylor approximation of $F$ around $p_{0}$ given by $F(x)=A x+\frac{1}{2 !} B(x, x)+\frac{1}{3 !} C(x, x, x)+\mathcal{O}\left(|x|^{4}\right)$. Assume that $A$ has a pair of purely imaginary eigenvalues $\pm \lambda i$. Let $q$ be the eigenvector of $A$ corresponding to the eigenvalue $\lambda i$, normalized so that $\bar{q} \cdot q=1$, where $\bar{q}$ is the conjugate vector of $q$. Let $p$ be the adjoint eigenvector such that $A^{T} p=-\lambda i p$ and $\bar{p} \cdot q=1$. If I denotes the $6 \times 6$ identity matrix, then

$$
\begin{array}{r}
\ell_{1}\left(p_{0}\right)=\frac{1}{2 \lambda} \operatorname{Re}\left(\bar{p} \cdot C(q, q, \bar{q})-2 \bar{p} \cdot B\left(q, A^{-1} B(q, \bar{q})\right)\right. \\
\left.+\bar{p} \cdot B\left(\bar{q},(2 \lambda i I-A)^{-1} B(q, q)\right)\right) .
\end{array}
$$

In our case the linear part of system (2) at the equilibrium $p_{0}$ is

$$
A=\left(\begin{array}{cccccc}
-1 & 0 & 0 & 0 & -2+\varepsilon \sigma & 0 \\
0 & -1 & 0 & 0 & 0 & -2+\varepsilon \sigma \\
0 & 0 & -1 & -2+\varepsilon \sigma & 0 & 0 \\
b \varepsilon & 0 & 0 & -b \varepsilon & 0 & 0 \\
0 & b \varepsilon & 0 & 0 & -b \varepsilon & 0 \\
0 & 0 & b \varepsilon & 0 & 0 & -b \varepsilon
\end{array}\right)+\mathcal{O}\left(\varepsilon^{2}\right)
$$

where

$\sigma=6 b+\frac{1}{(n-2)^{2}}\left(b^{2}(51-39 n)+b a 12 \sqrt[n]{\frac{n-2}{2}}-a^{2} \sqrt[n]{\left(\frac{n-2}{2}\right)^{2}}(n-1)\right)$.

We have that $A$ has an eigenvalue $\varepsilon \sqrt{3} b i+\mathcal{O}\left(\varepsilon^{2}\right)$. Now we compute the biand tri-linear functions $B$ and $C$. Considering the vector field $\left(f_{1}, f_{2}, f_{3}, f_{4}, f_{5}, f_{6}\right)$ associated to the differential system (2) we observe that all second and third 
derivatives vanishes except $\frac{\partial^{2} f_{1}}{\partial v^{2}}, \frac{\partial^{2} f_{2}}{\partial w^{2}}, \frac{\partial^{2} f_{3}}{\partial u^{2}}, \frac{\partial^{3} f_{1}}{\partial v^{3}}, \frac{\partial^{3} f_{2}}{\partial w^{3}}$ and $\frac{\partial^{3} f_{3}}{\partial u^{3}}$. Computing these derivatives, taking into account (4) and (5) we get that

$$
\begin{aligned}
& \frac{\partial^{2} f_{1}}{\partial v^{2}}\left(p_{0}\right)=\frac{\partial^{2} f_{2}}{\partial w^{2}}\left(p_{0}\right)=\frac{\partial^{2} f_{3}}{\partial u^{2}}\left(p_{0}\right)=\gamma, \\
& \frac{\partial^{3} f_{1}}{\partial v^{3}}\left(p_{0}\right)=\frac{\partial^{3} f_{2}}{\partial w^{3}}\left(p_{0}\right)=\frac{\partial^{3} f_{3}}{\partial u^{3}}\left(p_{0}\right)=\delta,
\end{aligned}
$$

where

$$
\begin{aligned}
\gamma= & -2(n-5) \sqrt[n]{\frac{n-2}{2}} \\
& +\varepsilon \sqrt[n]{\frac{2^{n-2}}{(n-2)^{n-2}}}\left(a(5 n-13)+3 b \sqrt[n]{\frac{2}{n-2}}\left(n^{2}-12 n+23\right)\right)+\mathcal{O}\left(\varepsilon^{2}\right),
\end{aligned}
$$

and

$$
\begin{aligned}
\delta= & -2 \sqrt[n]{\left(\frac{n-2}{2}\right)^{2}}\left(n^{2}-15 n+38\right) \\
& +\varepsilon \frac{2}{n-2} \sqrt[n]{\left(\frac{n-2}{2}\right)^{3}}\left(2 a\left(7 n^{2}-57 n+98\right)\right. \\
& \left.+3 b \sqrt[n]{\frac{2}{n-2}}\left(n^{3}-31 n^{2}+182 n-272\right)\right)+\mathcal{O}\left(\varepsilon^{2}\right)
\end{aligned}
$$

So, the bilinear function $B$ is given by

$$
B\left(\left(x_{1}, y_{1}, z_{1}, u_{1}, v_{1}, w_{1}\right),\left(x_{2}, y_{2}, z_{2}, u_{2}, v_{2}, w_{2}\right)\right)=\left(\gamma v_{1} v_{2}, \gamma w_{1} w_{2}, \gamma u_{1} u_{2}, 0,0,0\right),
$$

and the tri-linear function $C$ is given by the expression

$$
\begin{gathered}
C\left(\left(x_{1}, y_{1}, z_{1}, u_{1}, v_{1}, w_{1}\right),\left(x_{2}, y_{2}, z_{2}, u_{2}, v_{2}, w_{2}\right),\left(x_{3}, y_{3}, z_{3}, u_{3}, v_{3}, w_{3}\right)\right)= \\
\left(\delta v_{1} v_{2} v_{3}, \delta w_{1} w_{2} w_{3}, \delta u_{1} u_{2} u_{3}, 0,0,0\right) .
\end{gathered}
$$


Computing the normalized eigenvector $q$ of $A$, associated to the eigenvalue $\varepsilon \sqrt{3} b i+\mathcal{O}\left(\varepsilon^{2}\right)$, we obtain

$$
\begin{aligned}
q= & \left(\frac{1-\sqrt{3} i}{\sqrt{15}},-\frac{2}{\sqrt{15}}, \frac{1+\sqrt{3} i}{\sqrt{15}}, \frac{-1-\sqrt{3} i}{2 \sqrt{15}}, \frac{-1+\sqrt{3} i}{2 \sqrt{15}}, \frac{1}{\sqrt{15}}\right) \\
& +\varepsilon\left(\frac{-30(\sqrt{3}+i) b-(\sqrt{3}-3 i) \sigma}{30 \sqrt{5}}, \frac{60 b i+2 \sqrt{3} \sigma}{30 \sqrt{5}}, \frac{30(\sqrt{3}-i) b-(\sqrt{3}+3 i) \sigma}{30 \sqrt{5}},\right. \\
& \left.-\frac{(2 \sqrt{3}+6 i) \sigma}{30 \sqrt{5}},-\frac{(2 \sqrt{3}-6 i) \sigma}{30 \sqrt{5}}, \frac{4 \sqrt{3} \sigma}{30 \sqrt{5}}\right)+\mathcal{O}\left(\varepsilon^{2}\right) .
\end{aligned}
$$

The normalized adjoint eigenvector of the transpose matrix $A$ with the eigenvalue $-\varepsilon \sqrt{3} b i$ is

$$
\begin{aligned}
p= & \left(0,0,0, \frac{-\sqrt{15}-3 \sqrt{5} i}{6}, \frac{-\sqrt{15}+3 \sqrt{5} i}{6}, \frac{\sqrt{15}}{3}\right) \\
& +\varepsilon\left(\frac{(-5-5 \sqrt{3} i) b}{2 \sqrt{15}}, \frac{(-5+5 \sqrt{3} i) b}{2 \sqrt{15}}, \frac{5 b}{\sqrt{15}}, \frac{10 b+(1+\sqrt{3} i) \sigma}{\sqrt{15}},\right. \\
& \left.\frac{-5(1+\sqrt{3} i) b+(1-\sqrt{3} i) \sigma}{\sqrt{15}}, \frac{-5(1-\sqrt{3} i) b-2 \sigma}{\sqrt{15}}\right)+\mathcal{O}\left(\varepsilon^{2}\right) .
\end{aligned}
$$

According to Lemma 2 , in order to compute $\ell_{1}\left(p_{0}\right)$, we need to compute first $A^{-1}$ and $(2 \sqrt{3} b \varepsilon i I-A)^{-1}$. We have that $A^{-1}=\frac{1}{\varepsilon} A_{-1}+A_{0}+\varepsilon A_{1}+\mathcal{O}\left(\varepsilon^{2}\right)$,

$$
A_{-1}=\frac{1}{9 b}\left(\begin{array}{rrrrrr}
0 & 0 & 0 & 8 & 2 & -4 \\
0 & 0 & 0 & -4 & 8 & 2 \\
0 & 0 & 0 & 2 & -4 & 8 \\
0 & 0 & 0 & -1 & 2 & -4 \\
0 & 0 & 0 & -4 & -1 & 2 \\
0 & 0 & 0 & 2 & -4 & -1
\end{array}\right),
$$




$$
A_{1}=\frac{\sigma}{81 b}\left(\begin{array}{rrrrrr}
-12 b & 15 b & -12 b & -10 \sigma & 8 \sigma & -\sigma \\
-12 b & -12 b & 15 b & -\sigma & -10 \sigma & 8 \sigma \\
15 b & -12 b & -12 b & 8 \sigma & -\sigma & -10 \sigma \\
-12 b & 15 b & -12 b & -10 \sigma & 8 \sigma & -\sigma \\
-12 b & -12 b & 15 b & -\sigma & -10 \sigma & 8 \sigma \\
15 b & -12 b & -12 b & 8 \sigma & -\sigma & -10 \sigma
\end{array}\right) .
$$

and the expression of $(2 \sqrt{3} b \varepsilon i I-A)^{-1}$ is very large and we present it in the appendix.

The first, second and third terms of $\ell_{1}\left(p_{0}\right)$ given in Lemma 2 respectively are

$$
\begin{gathered}
\operatorname{Re}(\bar{p} \cdot C(q, q, \bar{q}))=\frac{1}{15} \sqrt[n]{\left(\frac{n-2}{2}\right)^{2}}\left(n^{2}-15 n+38\right) b \varepsilon+\mathcal{O}\left(\varepsilon^{2}\right) \\
\operatorname{Re}\left(-2 \bar{p} \cdot B\left(q, A^{-1} B(q, \bar{q})\right)\right)=-\frac{4}{45} \sqrt[n]{\left(\frac{n-2}{2}\right)^{2}}\left(n^{2}-10 n+25\right) b \varepsilon+\mathcal{O}\left(\varepsilon^{2}\right)
\end{gathered}
$$

and

$$
\operatorname{Re}\left(\bar{p} \cdot B\left(\bar{q},(2 \lambda i I-A)^{-1} B(q, q)\right)\right)=0+\mathcal{O}\left(\varepsilon^{2}\right) .
$$

Consequently we get

$$
\ell_{1}\left(p_{0}\right)=-\frac{2}{45 \sqrt{3}} \sqrt[n]{\left(\frac{n-2}{2}\right)^{2}}(n+7)(n-2)+\mathcal{O}(\varepsilon)
$$

As we said before $\ell_{1}\left(p_{0}\right)<0$ implies that we have a supercritical Hopf bifurcation at $\alpha=\alpha_{b i f}$, so there exists $\varepsilon_{0}>0$ such that for $\alpha_{b i f}<\alpha<$ $\alpha_{b i f}+\varepsilon_{0}$ the system (2) has a stable limit cycle.

\section{Conclusions}

The repressilator model is an implementation of an artificial oscillatory network used for studying the collections of interacting molecules in a cell. The model is given by a 6 -dimensional differential system. O. Buse, A. Kuznetsov and R. Pérez published two nice papers (see $[3,4]$ ) analysing a reduced system of dimension three. In this reduced system they show the existence of a supercritical Hopf bifurcation. Since the reduction is reasonable we may expect that such supercritical Hopf bifurcation must also occurs in the actual 6-dimensional differential system. We prove that this is the case. 


\section{Appendix}

The matrix $(2 \sqrt{3} b \varepsilon i I-A)^{-1}$ is given by

$$
(2 \sqrt{3} b \varepsilon i I-A)^{-1}=\left(\begin{array}{cccccc}
C_{11} & C_{12} & C_{13} & C_{14} & C_{15} & C_{16} \\
C_{21} & C_{22} & C_{23} & C_{24} & C_{25} & C_{26} \\
C_{31} & C_{32} & C_{33} & C_{34} & C_{35} & C_{36} \\
C_{41} & C_{42} & C_{43} & C_{44} & C_{45} & C_{46} \\
C_{51} & C_{52} & C_{53} & C_{54} & C_{55} & C_{56} \\
C_{61} & C_{62} & C_{63} & C_{64} & C_{65} & C_{66}
\end{array}\right),
$$

where $C_{i j}=A_{i j}+B_{i j}$, for $i, j=1,2, \ldots, 6$.

$$
\begin{aligned}
& A_{11}=\frac{1}{189}(213-16 i \sqrt{3}) \\
& A_{12}=\frac{2}{189}(-9+34 i \sqrt{3}) \\
& A_{13}=\frac{4}{189}(-15-4 i \sqrt{3}), \\
& A_{14}=\frac{4(12(\sqrt{3}-124 i) b \epsilon-(124 \sqrt{3}+3 i) \sigma \epsilon+72 \sqrt{3}+18 i)}{81(10 \sqrt{3}+27 i) b \epsilon} \\
& A_{15}=\frac{3156 \sqrt{3} b \epsilon+6144 i b \epsilon+512 \sqrt{3} \sigma \epsilon-789 i \sigma \epsilon-864 \sqrt{3}+666 i}{810 \sqrt{3} b \epsilon+2187 i b \epsilon}, \\
& A_{16}=\frac{4(12(50+87 i \sqrt{3}) b \epsilon+(261-50 i \sqrt{3}) \sigma \epsilon+18 i \sqrt{3}-225)}{81(27-10 i \sqrt{3}) b \epsilon}, \\
& A_{21}=\frac{4(12(50+87 i \sqrt{3}) b \epsilon+(261-50 i \sqrt{3}) \sigma \epsilon+18 i \sqrt{3}-225)}{81(27-10 i \sqrt{3}) b \epsilon}, \\
& A_{22}=\frac{1}{189}(213-16 i \sqrt{3}), \\
& A_{23}=\frac{1}{189}(213-16 i \sqrt{3}), \\
& A_{24}=\frac{4(12(50+87 i \sqrt{3}) b \epsilon+(261-50 i \sqrt{3}) \sigma \epsilon+18 i \sqrt{3}-225)}{81(27-10 i \sqrt{3}) b \epsilon}, \\
& A_{25}=\frac{4(12(50+87 i \sqrt{3}) b \epsilon+(261-50 i \sqrt{3}) \sigma \epsilon+18 i \sqrt{3}-225)}{81(27-10 i \sqrt{3}) b \epsilon}, \\
& A_{26}=\frac{4(12(50+87 i \sqrt{3}) b \epsilon+(261-50 i \sqrt{3}) \sigma \epsilon+18 i \sqrt{3}-225)}{81(27-10 i \sqrt{3}) b \epsilon}, \\
& A_{31}=\frac{4(12(50+87 i \sqrt{3}) b \epsilon+(261-50 i \sqrt{3}) \sigma \epsilon+18 i \sqrt{3}-225)}{81(27-10 i \sqrt{3}) b \epsilon}, \\
&
\end{aligned}
$$




$$
\begin{aligned}
& A_{32}=\frac{4(12(50+87 i \sqrt{3}) b \epsilon+(261-50 i \sqrt{3}) \sigma \epsilon+18 i \sqrt{3}-225)}{81(27-10 i \sqrt{3}) b \epsilon} \text {, } \\
& A_{33}=\frac{4(12(50+87 i \sqrt{3}) b \epsilon+(261-50 i \sqrt{3}) \sigma \epsilon+18 i \sqrt{3}-225)}{81(27-10 i \sqrt{3}) b \epsilon} \text {, } \\
& A_{34}=\frac{4(12(50+87 i \sqrt{3}) b \epsilon+(261-50 i \sqrt{3}) \sigma \epsilon+18 i \sqrt{3}-225)}{81(27-10 i \sqrt{3}) b \epsilon}, \\
& A_{35}=\frac{4(12(50+87 i \sqrt{3}) b \epsilon+(261-50 i \sqrt{3}) \sigma \epsilon+18 i \sqrt{3}-225)}{81(27-10 i \sqrt{3}) b \epsilon}, \\
& A_{36}=\frac{4(12(50+87 i \sqrt{3}) b \epsilon+(261-50 i \sqrt{3}) \sigma \epsilon+18 i \sqrt{3}-225)}{81(27-10 i \sqrt{3}) b \epsilon}, \\
& A_{41}=\frac{1}{189}(9-34 i \sqrt{3}) \text {, } \\
& A_{42}=\frac{2}{189}(15+4 i \sqrt{3}) \text {, } \\
& A_{43}=\frac{4}{189}(-3+2 i \sqrt{3}) \text {, } \\
& A_{44}=-\frac{912 \sqrt{3} b \epsilon+480 i b \epsilon+40 \sqrt{3} \sigma \epsilon-228 i \sigma \epsilon-432 \sqrt{3}+333 i}{810 \sqrt{3} b \epsilon+2187 i b \epsilon}, \\
& A_{45}=\frac{1188 \sqrt{3} b \epsilon-984 i b \epsilon-82 \sqrt{3} \sigma \epsilon-297 i \sigma \epsilon+36 \sqrt{3}+450 i}{810 \sqrt{3} b \epsilon+2187 i b \epsilon}, \\
& A_{46}=\frac{4(12(\sqrt{3}+44 i) b \epsilon+(44 \sqrt{3}-3 i) \sigma \epsilon-9(4 \sqrt{3}+i))}{81(10 \sqrt{3}+27 i) b \epsilon}, \\
& A_{51}=\frac{4}{189}(-3+2 i \sqrt{3}) \text {, } \\
& A_{52}=\frac{4}{189}(-3+2 i \sqrt{3}) \text {, } \\
& A_{53}=\frac{2}{189}(15+4 i \sqrt{3}) \text {, } \\
& A_{54}=\frac{4(12(\sqrt{3}+44 i) b \epsilon+(44 \sqrt{3}-3 i) \sigma \epsilon-9(4 \sqrt{3}+i))}{81(10 \sqrt{3}+27 i) b \epsilon}, \\
& A_{55}=-\frac{912 \sqrt{3} b \epsilon+480 i b \epsilon+40 \sqrt{3} \sigma \epsilon-228 i \sigma \epsilon-432 \sqrt{3}+333 i}{810 \sqrt{3} b \epsilon+2187 i b \epsilon}, \\
& A_{56}=\frac{1188 \sqrt{3} b \epsilon-984 i b \epsilon-82 \sqrt{3} \sigma \epsilon-297 i \sigma \epsilon+36 \sqrt{3}+450 i}{810 \sqrt{3} b \epsilon+2187 i b \epsilon}, \\
& A_{61}=\frac{2}{189}(15+4 i \sqrt{3}) \text {, } \\
& A_{62}=\frac{4}{189}(-3+2 i \sqrt{3}) \text {, } \\
& A_{63}=\frac{1}{189}(9-34 i \sqrt{3}) \text {, } \\
& A_{64}=\frac{1188 \sqrt{3} b \epsilon-984 i b \epsilon-82 \sqrt{3} \sigma \epsilon-297 i \sigma \epsilon+36 \sqrt{3}+450 i}{810 \sqrt{3} b \epsilon+2187 i b \epsilon},
\end{aligned}
$$




$$
\begin{aligned}
& A_{65}=\frac{1188 \sqrt{3} b \epsilon-984 i b \epsilon-82 \sqrt{3} \sigma \epsilon-297 i \sigma \epsilon+36 \sqrt{3}+450 i}{810 \sqrt{3} b \epsilon+2187 i b \epsilon}, \\
& A_{66}=\frac{1188 \sqrt{3} b \epsilon-984 i b \epsilon-82 \sqrt{3} \sigma \epsilon-297 i \sigma \epsilon+36 \sqrt{3}+450 i}{810 \sqrt{3} b \epsilon+2187 i b \epsilon}, \\
& B_{11}=\frac{2 \epsilon((-4800-5921 i \sqrt{3}) b+(-362+316 i \sqrt{3}) \sigma)}{3969}, \\
& B_{12}=\frac{\epsilon(8(215 \sqrt{3}+42 i) b-(2 \sqrt{3}+259 i) \sigma)}{81(4 \sqrt{3}+i)}, \\
& B_{13}=\frac{\epsilon(8(215 \sqrt{3}+42 i) b-(2 \sqrt{3}+259 i) \sigma)}{81(4 \sqrt{3}+i)}, \\
& B_{14}=\frac{2 \epsilon}{81(2 \sqrt{3}-3 i)^{3} b}\left(96(444 \sqrt{3}-131 i) b^{2}-52(41 \sqrt{3}+324 i) b \sigma\right. \\
& \left.+(-516 \sqrt{3}+271 i) \sigma^{2}\right), \\
& B_{15}=\frac{\epsilon}{81(2 \sqrt{3}-3 i)^{3} b}\left(24(-1760 \sqrt{3}+5807 i) b^{2}+2(9247 \sqrt{3}+5952 i) b \sigma\right. \\
& \left.+8(14 \sqrt{3}-215 i) \sigma^{2}\right), \\
& B_{16}=\frac{\epsilon}{81(2 \sqrt{3}-3 i)^{3} b}\left(-48(1058 \sqrt{3}+2825 i) b^{2}+16(-1021 \sqrt{3}\right. \\
& \left.+1362 i) b \sigma+(758 \sqrt{3}+1259 i) \sigma^{2}\right) \text {, } \\
& B_{21}=\frac{4 \epsilon((-146 \sqrt{3}+528 i) b+(32 \sqrt{3}+23 i) \sigma)}{81(4 \sqrt{3}+i)}, \\
& B_{22}=\frac{2 \epsilon((-4800-5921 i \sqrt{3}) b+(-362+316 i \sqrt{3}) \sigma)}{3969}, \\
& B_{23}=\frac{\epsilon(8(215 \sqrt{3}+42 i) b-(2 \sqrt{3}+259 i) \sigma)}{81(4 \sqrt{3}+i)}, \\
& B_{24}=\frac{\epsilon}{81(2 \sqrt{3}-3 i)^{3} b}\left(-48(1058 \sqrt{3}+2825 i) b^{2}+16(-1021 \sqrt{3}\right. \\
& \left.+1362 i) b \sigma+(758 \sqrt{3}+1259 i) \sigma^{2}\right), \\
& B_{25}=\frac{2 \epsilon}{81(2 \sqrt{3}-3 i)^{3} b}\left(96(444 \sqrt{3}-131 i) b^{2}\right. \\
& \left.-52(41 \sqrt{3}+324 i) b \sigma+(-516 \sqrt{3}+271 i) \sigma^{2}\right), \\
& B_{26}=\frac{\epsilon}{81(2 \sqrt{3}-3 i)^{3} b}\left(24(-1760 \sqrt{3}+5807 i) b^{2}+2(9247 \sqrt{3}\right. \\
& \left.+5952 i) b \sigma+8(14 \sqrt{3}-215 i) \sigma^{2}\right) \text {, } \\
& B_{31}=\frac{\epsilon(8(215 \sqrt{3}+42 i) b-(2 \sqrt{3}+259 i) \sigma)}{81(4 \sqrt{3}+i)}, \\
& B_{32}=\frac{2 \epsilon((-4800-5921 i \sqrt{3}) b+(-362+316 i \sqrt{3}) \sigma)}{3969}, \\
& B_{33}=\frac{2 \epsilon((-4800-5921 i \sqrt{3}) b+(-362+316 i \sqrt{3}) \sigma)}{3969},
\end{aligned}
$$




$$
\begin{aligned}
& B_{34}=\frac{\epsilon}{81(2 \sqrt{3}-3 i)^{3} b}\left(24(-1760 \sqrt{3}+5807 i) b^{2}+2(9247 \sqrt{3}\right. \\
& \left.+5952 i) b \sigma+8(14 \sqrt{3}-215 i) \sigma^{2}\right), \\
& B_{35}=\frac{\epsilon}{81(2 \sqrt{3}-3 i)^{3} b}\left(-48(1058 \sqrt{3}+2825 i) b^{2}+16(-1021 \sqrt{3}\right. \\
& \left.+1362 i) b \sigma+(758 \sqrt{3}+1259 i) \sigma^{2}\right), \\
& B_{36}=\frac{2 \epsilon}{81(2 \sqrt{3}-3 i)^{3} b}\left(96(444 \sqrt{3}-131 i) b^{2}-52(41 \sqrt{3}+324 i) b \sigma\right. \\
& \left.+(-516 \sqrt{3}+271 i) \sigma^{2}\right), \\
& B_{41}=\frac{2 \epsilon(-259 \sqrt{3} b+6 i b+8 \sqrt{3} \sigma+22 i \sigma)}{81(4 \sqrt{3}+i)}, \\
& B_{42}=-\frac{\epsilon(8(-23 \sqrt{3}+96 i) b+(40 \sqrt{3}+19 i) \sigma)}{81(4 \sqrt{3}+i)}, \\
& B_{43}=\frac{4 \epsilon(2(35 \sqrt{3}+54 i) b+(6 \sqrt{3}-13 i) \sigma)}{81(4 \sqrt{3}+i)}, \\
& B_{44}=\frac{2 \epsilon}{81(2 \sqrt{3}-3 i)^{3} b}\left(96(14 \sqrt{3}-215 i) b^{2}-52(53 \sqrt{3}+6 i) b \sigma\right. \\
& \left.+(2 \sqrt{3}+259 i) \sigma^{2}\right) \\
& B_{45}=\frac{\epsilon}{81(2 \sqrt{3}-3 i)^{3} b}\left(24(758 \sqrt{3}+1259 i) b^{2}+2(1627 \sqrt{3}\right. \\
& \left.-3810 i) b \sigma-8(32 \sqrt{3}+23 i) \sigma^{2}\right), \\
& B_{46}=\frac{\epsilon}{81(2 \sqrt{3}-3 i)^{3} b}\left(48(-516 \sqrt{3}+271 i) b^{2}+16(131 \sqrt{3}\right. \\
& \left.+576 i) b \sigma+(252 \sqrt{3}-253 i) \sigma^{2}\right), \\
& B_{51}=\frac{4 \epsilon(2(35 \sqrt{3}+54 i) b+(6 \sqrt{3}-13 i) \sigma)}{81(4 \sqrt{3}+i)}, \\
& B_{52}=\frac{2 \epsilon(-259 \sqrt{3} b+6 i b+8 \sqrt{3} \sigma+22 i \sigma)}{81(4 \sqrt{3}+i)}, \\
& B_{53}=-\frac{\epsilon(8(-23 \sqrt{3}+96 i) b+(40 \sqrt{3}+19 i) \sigma)}{81(4 \sqrt{3}+i)}, \\
& B_{54}=\frac{\epsilon}{81(2 \sqrt{3}-3 i)^{3} b}\left(48(-516 \sqrt{3}+271 i) b^{2}+16(131 \sqrt{3}\right. \\
& \left.+576 i) b \sigma+(252 \sqrt{3}-253 i) \sigma^{2}\right), \\
& B_{55}=\frac{2 \epsilon}{81(2 \sqrt{3}-3 i)^{3} b}\left(96(14 \sqrt{3}-215 i) b^{2}-52(53 \sqrt{3}+6 i) b \sigma\right. \\
& \left.+(2 \sqrt{3}+259 i) \sigma^{2}\right) \\
& B_{56}=\frac{\epsilon}{81(2 \sqrt{3}-3 i)^{3} b}\left(24(758 \sqrt{3}+1259 i) b^{2}+2(1627 \sqrt{3}\right. \\
& \left.-3810 i) b \sigma-8(32 \sqrt{3}+23 i) \sigma^{2}\right),
\end{aligned}
$$




$$
\begin{aligned}
& B_{61}=-\frac{\epsilon(8(-23 \sqrt{3}+96 i) b+(40 \sqrt{3}+19 i) \sigma)}{81(4 \sqrt{3}+i)}, \\
& B_{62}=\frac{4 \epsilon(2(35 \sqrt{3}+54 i) b+(6 \sqrt{3}-13 i) \sigma)}{81(4 \sqrt{3}+i)}, \\
& B_{63}=\frac{4 \epsilon(2(35 \sqrt{3}+54 i) b+(6 \sqrt{3}-13 i) \sigma)}{81(4 \sqrt{3}+i)}, \\
& B_{64}=\frac{\epsilon}{81(2 \sqrt{3}-3 i)^{3} b}\left(24(758 \sqrt{3}+1259 i) b^{2}+2(1627 \sqrt{3}\right. \\
& \left.-3810 i) b \sigma-8(32 \sqrt{3}+23 i) \sigma^{2}\right), \\
& B_{65}=\frac{\epsilon}{81(2 \sqrt{3}-3 i)^{3} b}\left(48(-516 \sqrt{3}+271 i) b^{2}+16(131 \sqrt{3}\right. \\
& \left.+576 i) b \sigma+(252 \sqrt{3}-253 i) \sigma^{2}\right) \text { and } \\
& B_{66}=\frac{2 \epsilon}{81(2 \sqrt{3}-3 i)^{3} b}\left(96(14 \sqrt{3}-215 i) b^{2}-52(53 \sqrt{3}+6 i) b \sigma\right. \\
& \left.+(2 \sqrt{3}+259 i) \sigma^{2}\right) \text {. }
\end{aligned}
$$

\section{ACKNOWLEDGMents}

The first author is supported by FAPESP-BRAZIL grant 2007/068965, CAPES-PROCAD-BRAZIL grant 035/2007 and CNPQ-BRAZIL grant 472787/2009-8. The second author is supported by MINECO/FEDER grant MTN2008-03437, by AGAUR grant number 2009SGR-0410, by ICREA Academia and by FP7-PEOPLE-2012-IRSES 316338 and 318999. Both authors are also supported by the joint project CAPES-MECD grant PHB-20090025-PC. The first author would like to thanks for the hospitality at the Departament de Matemàtiques of Universitat Autònoma de Barcelona where this work was done.

\section{REFERENCES}

[1] Atkinson, M., Savageau, M., Myers, J. and Ninfa, A. [2003] "Development of genetic circuitry exhibiting toggle switch or oscillatory behavior in escherichia coli", Cell. 113, pp. 597-607.

[2] Barkai, N. and Leibler, S. [2000] "Circadian clocks limited by noise", Nature 403, pp. $267-268$.

[3] Buse, O., Kuznetsov, A. and Pérez, R. [2009] "Existence of limit cycles in the repressilator equations", Int. J. Bifurcation Chaos Appl. Sci. Eng. 19, pp. 4097-4106.

[4] Buse, O., Kuznetsov, A. and Pérez, R. [2010] "Dynamical properties of the repressilator model", Phys. Review E 81, pp. 066206. 
[5] Elowitz, M. and Leibler, S. [2000] "A synthetic oscillatory network of transcriptional regulators", Nature 403, pp. 335-338.

[6] Garcia-Ojalvo, J., Elowitz, M. B. and Strogatz, S. H. [2004] "Modeling a synthetic multicellular clock: Repressilators coupled by quorum sensing", PNAS 101, no. 30, pp. $10955-10960$.

[7] Hasty, J., Isaacs, F., Dolnik, M., McMillen, D. and Collins, J. J. [2001] "Designer gene networks: Toward fundamental cellular control", Chaos 11, pp. 207-220.

[8] Kuznetsov, A., Kaern, M. and Kopell, N. [2004] "Synchrony in a population of hysteresis-based genetic oscillators", SIAM J. Appl. Math. 65, pp. 392-425.

[9] Kuznetsov, Y. [2004] Elements of applied bifurcation theory, Applied Mathematical Sciences, Vol. 112, Springer-Verlag, New York.

[10] Strelkowa, N. and Barahona, M. [2011] "Transient dynamics around unstable periodic orbits in the generalized repressilator model", Chaos 21, pp. 023104.

[11] Tokuda, I. T., Wagemakers, A. and Sanjuán, M. A. F. [2010] "Predicting thesynchronization of a network of electronic repressilators", International Journal of Bifurcation and Chaos 20, no. 6, pp. 1751-1760.

1 Departamento de Matemática - ibilce-UnesP, Rua C. Colombo, 2265, CEP 15054-000 S. J. Rio Preto, SÃo Paulo, Brazil

E-mail address: buzzi@ibilce.unesp.br

2 Departament de Matemàtiques - Universitat Autònoma de Barcelona, 08193 Bellaterra, Barcelona, Spain

E-mail address: jllibre@mat.uab.cat 